\title{
Incidental Diagnosis of Primary Tuberculosis of the Palatine Tonsil in a Patient With Nasal Obstruction and Snoring
}

\author{
Jinsoon Chang ${ }^{\mathrm{a}}$, Mun Cheol Kim ${ }^{\mathrm{b}, \mathrm{c}}$
}

\begin{abstract}
Tuberculosis (TB) is caused by Mycobacterium tuberculosis and most commonly occurs in the lung. Only $10-15 \%$ of all cases of tuberculosis occur outside the respiratory system. Whereas secondary tuberculosis of the tonsil, where there is sputum-positive pulmonary TB, has been well documented, primary tuberculosis of the tonsil without pulmonary TB is rare, and only a few cases have been reported up to now. However, primary palatine tonsillar tuberculosis without either lung or cervical lymph node involvement has not yet been reported. The authors present a case of incidental diagnosis of primary palatine tonsillar TB following postoperative pathologic examination in an otherwise healthy 35-year-old woman who underwent bilateral tonsillectomy with endoscopic sinus surgery due to nasal obstruction and snoring. The patient received anti-tuberculosis medical treatment after tonsillectomy and was successfully treated.
\end{abstract}

Keywords: Tuberculosis; Palatine tonsil; Tonsillectomy

\section{Introduction}

Tuberculosis (TB) is an airborne disease that primarily affects the lung, but can also affect other parts of the body. Regardless of its site of infection, tuberculosis remains widespread across the world and is especially prevalent in Southeast Asia and Africa. Primary tuberculosis involving the head and neck area is extremely rare and constitutes only $2-6 \%$ of the cases of tuberculosis outside the lungs and $0.1-1 \%$ of all forms of tuberculosis [1]. Primary tonsillar tuberculosis is an even rarer clinical entity and is often mistaken for tonsillar malignancy [2]. The authors present a case of primary tonsillar tuberculosis in a female adult who suffered from nasal obstruction and snoring. We performed endoscopic sinus surgery and tonsillectomy to

Manuscript submitted December 27, 2018, accepted January 4, 2019

aDepartment of Otorhinolaryngology-Head and Neck Surgery, Seoul Paik Hospital, Inje University College of Medicine, Seoul, Korea

${ }^{b}$ Department of Anesthesiology and Pain Medicine, Sanggye Paik Hospital, Inje University College of Medicine, Seoul, Korea

${ }^{\mathrm{c}}$ Corresponding Author: Mun Cheol Kim, Dongil-ro 1342, Nowon-gu, Seoul 01757, Korea. Email: daumkmcan@hanmail.net improve her naso- and oropharyngeal airway. Tonsillar tuberculosis was pathologically confirmed after tonsillectomy. We report this case to highlight that primary tonsillar tuberculosis can exist without symptoms and signs and to draw attention to latent tonsillar tuberculosis.

\section{Case Report}

A 35-year-old female patient was hospitalized for surgery for sinusitis and snoring. She had intermittent sore throat and snoring without any definitive treatment. There was a previous history of removal of a right nasal polyp, but there was no known family history or other specific findings. Physical examination revealed bilateral palatal tonsillar hypertrophy of Friedman stage III. There were no pathological findings in the teeth or other parts of the mouth.

In addition, no cervical lymph nodes or masses were palpable and the patient's general condition was healthy. Chest $\mathrm{X}$-ray and electrocardiogram results showed no abnormal findings (Fig. 1).

Preoperative sinus computed tomography showed right sinusitis and bilateral tonsillar hypertrophy (Fig. 2). Right endo-

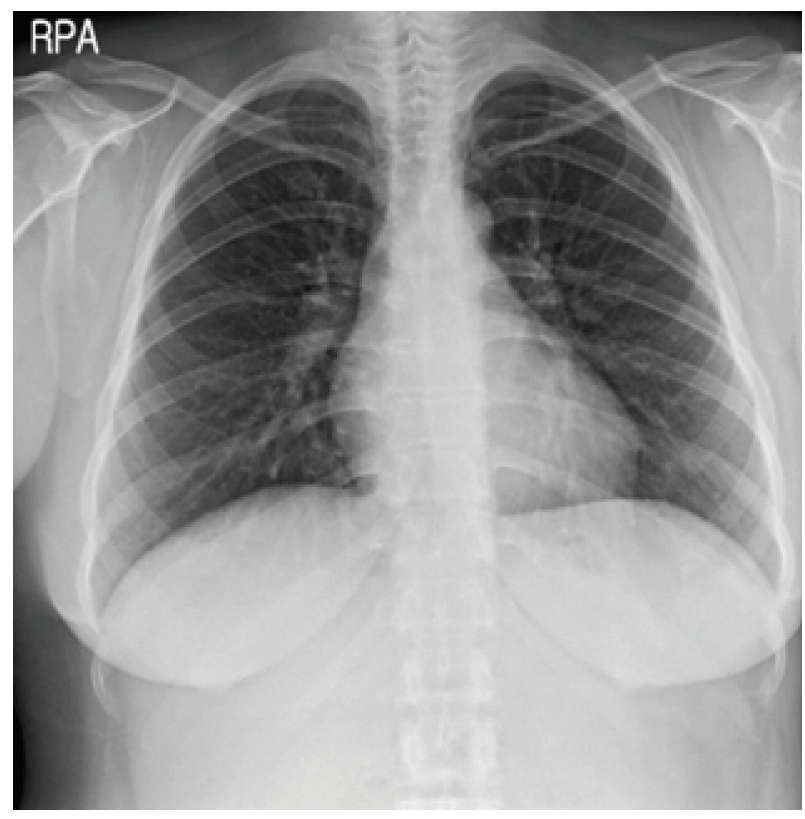

Figure 1. Chest X-ray was within normal limits. 


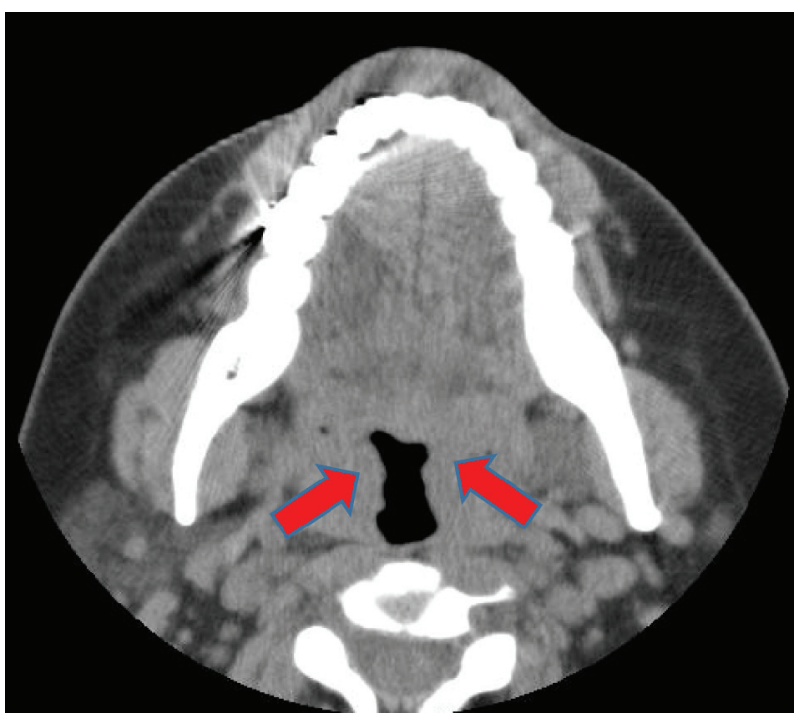

Figure 2. The preoperative paranasal sinus computed tomography image. Bilateral tonsillar hypertrophy (red arrow) is observed. There were no other specific findings.

scopic sinus surgery and bilateral tonsillectomy were planned and performed under general anesthesia. During the operation, the detachment of bilateral enlarged tonsils from the tonsillar bed was relatively straightforward and unremarkable. No specific findings, such as different tissue consistency or contour, were suspected of either tonsil other than chronic bilateral tonsillar hypertrophy. Postoperative pathologic examination revealed Langerhans-type giant cells with central necrosis apparent on hematoxylin and eosin staining (Fig. 3). In addition, the Tb-PCR identification test provided positive findings. However, an additional acid-fast bacilli (AFB) test provided negative findings. Because of the lack of history of tuberculosis, negative family history, and chest X-ray findings which showed that pulmonary tuberculosis was absent, we made a primary diagnosis of palatine tonsillar tuberculosis. HERZ's 4-tube anti-tuberculosis therapy was provided for a total duration of 9 months. Twelve months after the operation, the patient was regularly followed up with an unremarkable recovery.

\section{Discussion}

Mycobacterium is known to affect primarily the lungs but can affect extrapulmonary organs as well. Sites of infection other than the lung account for about $25 \%$ of cases of tuberculosis. The most common site other than the lung is the lymph nodes [3]. In the upper respiratory tract, the most common site of involvement is the larynx. Although tuberculosis of the oral cavity is uncommon, the tongue and palate are more prevalent sites, whereas the tonsil is rarely involved with an incidence of less than 5\% [4]. The incidence of secondary tonsillar tuberculosis in a patient with pulmonary tuberculosis ranges from $40-80 \%$, and for those cases whose tuberculosis is not seen on chest Xray examination, it is said to be histologically proven in about $0.2-6.2 \%[5]$. The main route of primary tonsillar tuberculosis

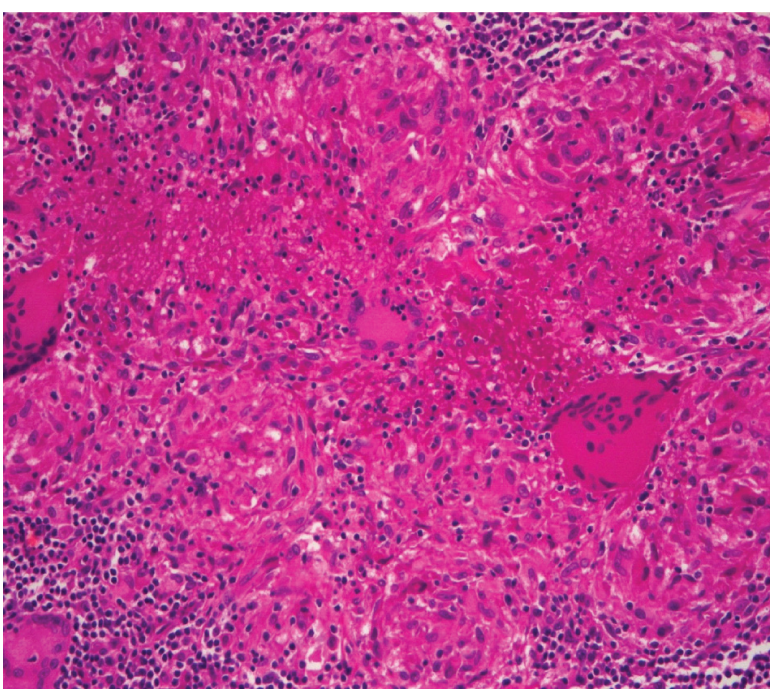

Figure 3. Histopathological examination revealed presence of tubercular granuloma characterized by epithelioid cells, Langerhans-type giant cells, and mononuclear inflammatory cells (H\&E, $200 \times)$.

is infection by contact with material containing tubercle bacilli. It is believed that the causative bacteria are latent in Waldeyer's ring and cause opportunistic infection [4]. It is more common in susceptible adults with chronic alcoholism and uncontrolled diabetes, and other general immune-suppressing conditions may cause tuberculosis in areas other than the lung. Although it is still prevalent, the incidence of TB is known to have gradually decreased in recent years in Korea due to the development of preventive measures against tuberculosis, advancement of anti-tuberculosis treatment, improvement of socioeconomic status, and improvement of systemic physical conditions and environmental cleaning. Any difference in risk by age or sex was not established for tonsillar tuberculosis, but poor oral hygiene, history of dental extraction, and leukoplakia are related to the incidence of the disease [6]. In the same context, rising incidence rates in developing and developed countries may be related to immune deficiency, inadequate nutritional status, and increasing resistance to medical therapy [7]. Symptoms of tonsillar tuberculosis include pain, difficulty in swallowing, foreign body sensation, and cervical lymphadenopathy in onethird of patients. These clinical factors along with physical findings, including a past history of tuberculosis, and chest X-ray findings, are pertinent in making the diagnosis [8].

Unfortunately, the present patient did not exhibit any of these symptoms or factors suggesting tonsillar tuberculosis. Therefore, the test was not performed preoperatively. A definite diagnosis can be confirmed using pathologic findings that demonstrate the presence of AFB upon staining. The treatment of tonsillar tuberculosis consists mainly of anti-tuberculosis therapy after tonsillectomy, cleansing of the local area, and strengthening of the immune system against further infection. Although the period of anti-tuberculosis treatments for primary tonsillar tuberculosis has not been well established, it has been reported that treatment strategies based on therapy paralleling pulmonary tuberculosis are generally effective [9]. However, in cases of extrapulmonary tuberculosis, such as tuberculo- 
sis lymphadenitis, joints, and bone marrow tuberculosis, more long-term anti-tuberculosis therapy is required [10]. Even if the number of patients with tuberculosis is continuously decreasing, it seems reasonable that not only the treatment of tuberculosis but also the treatment of high-risk patients with possibly latent tuberculosis needs to be considered more thoroughly due to an increasing rate of immunocompromised patients secondary to aging and advancements in medical treatment. Therefore, the possibility of latent tonsillar tuberculosis when treating patients with bilateral hypertrophy should be taken into consideration and clinicians should have a high index of suspicion for the diagnosis of latent tuberculosis when considering the treatment plan. Based on our experience, we conclude that in patients with chronic tonsillitis or tonsillar hypertrophy, more in-depth examination should be performed to distinguish other diseases from tonsillar tuberculosis, especially in patients having predisposing factors. Histopathologic examination should be confirmed after surgery even if the tonsils retain their normal appearance.

In conclusion, primary tonsil tuberculosis is rare and may exist without any signs or symptoms suggestive of tuberculosis; thus, care should be taken in making a diagnosis and formulating a treatment plan.

\section{Reference}

1. Chavolla R, Dolci GF, Hernandez JFM, Ysunza A, Cue- vas B, Lazos M, et al. Primary tuberculosis of the tonsil. Int J Pediatr Otorhinolaryngol. 2008;72(8):1159-1165.

2. Gulati A, Kaushik R, Sharma J. Primary tuberculosis of the tonsil masquerading as carcinoma. Indian J Pathol Microbiol. 2006;49(2):312-313.

3. Das A, Das SK, Pandit S, Basuthakur S. Tonsillar tuberculosis: a forgotten clinical entity. J Family Med Prim Care. 2015;4(1):124-126.

4. Prasad P, Bhardwaj M. Primary tuberculosis of tonsils: a case report. Case Rep Med. 2012;2012:120382.

5. Ock HN, Chung SD, Moon SM, Lee SC. A case of primary nasal and pharyngeal tuberculosis. Korean J Otolaryngol 1974; 7:365-368

6. Karki S, Karki D. Tonsillar tuberculosis. J Pathol Nepal. 2016;6:48-50.

7. Gupta KB, Tandon S, Jaswal TS, Singh S. Tuberculosis of tonsil with unsusal presentation. Ind J Tub. 2001;48:223224.

8. Wang CW, Chang CF. The association of secondary tonsillar tuberculosis with asymptomatic pulmonary focus. J of Infectious Diseases and Treatment. 2016;2:1-3.

9. Harding SM, Bailey WC. Chemotherapy of tuberculosis. In: Schlossberg D, editor. Tuberculosis. 3rd Ed. SpringerVerlag: New York, 1994; pp 69-88.

10. Friedman B, Kapur VN. Newer knowledge of chemotherapy in the treatment of tuberculosis of bones and joints. Clin Orthop Relat Res. 1973;97:5-15. 